At the Leiden Early Arthritis Clinic from 19931999, 285 patients fulfilled the criteria for RA after 1 year's follow-up. Destruction of joints (hands and feet) was scored radiographically using the Sharp-van der Heijde method at baseline and yearly thereafter. The extremes of disease course were compared: those patients who had achieved American College of Rheumatology-defined sustained remission (28 patients) and the same number of patients with the most severe small joint destruction (at 1 year's follow-up). Comparison revealed an association between arthritis of the large joints (especially the knee) at first presentation and a severe RA course. In the whole group $(n=285)$, presence of knee arthritis and total number of inflamed joints were both independently associated with the subsequent severity of RA. Interestingly, patients presenting with knee arthritis had increased levels of C-reactive protein; the level of which is a predictor of RA disease severity.

The patients included in this study offered an excellent opportunity to investigate factors associated with the natural course of RA. As knee involvement is physically assessed at the first clinical visit, the results of this study offer doctors an early guide to assessing potential outcome.

Original article Linn-Rasker SP et al. (2007) Arthritis of the large joints - in particular, the knee-at first presentation is predictive for a high level of radiological destruction of the small joints in rheumatoid arthritis. Ann Rheum Dis 66: 646-650

\section{Temperature and change in atmospheric pressure affect pain severity in patients with $O A$}

The suggestion that pain severity in individuals with arthritis is affected by weather conditions has not been proved conclusively, owing to the difficulty of studying this phenomenon without considerable bias influencing the results. McAlindon and colleagues investigated the effect of both ambient weather conditions and changes in weather conditions on pain severity in patients with knee osteoarthritis (OA).

The investigators enrolled 200 patients who had previously participated in an online trial that studied the effect of glucosamine on the symptoms of knee OA. Participants (mean age 60 years, 64\% women) were dispersed across the US, and $96 \%$ of them lived within 1 mile of a weather station from which meteorological data (including average daily temperature, barometric pressure, precipitation and relative humidity) were obtained for each day of the study. The pain subscale of the WOMAC questionnaire was completed by participants, via the internet, at baseline and every 2 weeks for a total of seven assessments.

Several statistical models and adjustment of results to include factors such as age, sex, and NSAID use showed that there was a significant, positive correlation between WOMAC pain score and the difference in pressure between the day before and the day of the pain report (coefficient $=1.14, P=0.02$ ), and a significant, negative correlation between WOMAC pain score and ambient temperature (coefficient $=-0.01, P=0.004$ ). . No interactions between temperature and pressure change had any effect on the results.

The authors conclude that, although the effects of pressure change and ambient temperature on pain are small in terms of clinical importance, the therapeutic relevance of their results warrants further study.

Original article McAlindon T et al. (2007) Changes in barometric pressure and ambient temperature influence osteoarthritis pain. Am J Med 120: 429-434 